PROCEEDINGS OF THE AMERICAN MATHEMATICAL SOCIETY

Volume 124, Number 1, January 1996

\title{
ON SEPARATION PROPERTIES OF FINITE DIMENSIONAL COMPACT CONVEX SETS
}

\author{
JOÃO F. QUEIRÓ AND EDUARDO M. SÀ \\ (Communicated by Dale Alspach)
}

\begin{abstract}
In a real finite-dimensional vector space, we study families of sets such that every compact convex set in the space is the intersection of all members of the family that contains it.
\end{abstract}

\section{INTRODUCTION}

In this paper we consider convex sets in a real vector space. We shall restrict ourselves to the finite-dimensional case (or, without loss of generality, to $\mathbb{R}^{n}$ ), although some of our comments and results hold in more general topological vector spaces.

The kind of question we are interested in is the following: for a given type of convex sets, which families of sets are such that every set of the prescribed type is equal to the intersection of all members of the family that contain it? This is a simple matter for closed convex sets, and our purpose in the paper is to answer it for compact convex sets.

For closed convex sets, a well-known theorem of Minkowski says that every such set is the intersection of closed half-spaces. Are there other families of sets satisfying this property? The answer is essentially negative, as shown by the following result.

Proposition 1.1. Let $\mathcal{S}$ be a family of closed convex sets, such that every closed convex set in the space is the intersection of a subfamily of $\mathcal{S}$. Then every closed convex set in the space is the intersection of half-spaces belonging to $\mathcal{S}$.

Proof. If a closed convex set which is not the whole space contains a half-space, it must be a half-space itself. It follows that every closed half-space is the intersection of half-spaces belonging to $\mathcal{S}$. The result now follows from Minkowski's theorem.

We now turn to compact convex sets, and restate our problem: we want to know which families $\mathcal{S}$ of closed convex sets satisfy the property

every compact convex set in the space

is the intersection of a subfamily of $\mathcal{S}$.

Received by the editors April 20, 1992.

1991 Mathematics Subject Classification. Primary 52A20.

The authors were partially supported by Instituto Nacional de Investigação Científica and Junta Nacional de Investigação Científica e Tecnológica. 
To describe one example of such a family, denote by $B_{e}$ the unit ball, $B_{e}:=\{x \in$ $\left.\mathbb{R}^{n}:\|x\| \leq 1\right\}$, where $\|\cdot\|$ is the standard Euclidean norm of $\mathbb{R}^{n}$. Then, the family of all closed balls, namely

$$
\mathcal{S}_{e}:=\left\{r B_{e}+a: r>0 \text { and } a \in \mathbb{R}^{n}\right\},
$$

satisfies (1). In fact, even the family of balls $r B_{e}+a$ with $(r, a)$ running over a dense subset of $\mathbb{R}_{+} \times \mathbb{R}^{n}$ satisfies (1).

To simplify our discussion, we shall study families $\mathcal{S}$ which are closed under translations and positive homotheties, i.e. such that

$$
\alpha C+w \in \mathcal{S}, \quad \text { for any } C \in \mathcal{S}, w \in \mathbb{R}^{n} \text { and } \alpha>0 .
$$

We shall give a necessary and sufficient condition for such a family to satisfy property (1). Our condition involves the set of outward normals to each $C \in \mathcal{S}$ at points of differentiability of the boundary of $C$. Our main result cannot be called an unexpected one, in view of its appealing, intuitively acceptable geometrical meaning and flavour.

The kind of problem we are dealing with calls for appropriate separation theorems. In the literature we found several such theorems which use separating convex surfaces other than hyperplanes: for example, separation by spherical surfaces, cylinders and parallelotopes have been considered in $[2,3,4]$ for a different type of problem. Here, we are using very general separating surfaces, and prove general separation theorems under appropriate differentiability conditions, and then show that these conditions are best possible in a certain sense. For a related reasoning, see the proof of [1, Theorem 2.1].

The symbol $\langle x \mid y\rangle$ denotes the usual inner product of $x$ and $y$ in $\mathbb{R}^{n}$. Our reference for results and notation concerning convex sets and functions, subdifferentials, separation, etc., is [5].

\section{RESUlts}

In this article, $\mathcal{F}$ denotes a family of functionals, $f: \mathbb{R}^{n} \rightarrow \mathbb{R}_{+}$, which are convex and positively homogeneous (i.e. $f(\lambda x)=\lambda f(x)$, for any $x \in \mathbb{R}^{n}$ and $\lambda \geq 0$ ). We shall always assume that $\mathcal{F}$ is a cone, i.e. it satisfies the property

$$
\alpha f \in \mathcal{F}, \quad \text { for any } f \in \mathcal{F} \text { and } \alpha \in \mathbb{R}_{+} \text {. }
$$

The set $\left\{x \in \mathbb{R}^{n}: f(x) \leq 1\right\}$ will be denoted by $B_{f}$. Obviously, $B_{f}$ is a convex, closed neighborhood of the origin.

We define $\nabla(\mathcal{F})$ as the set of all $\gamma \in \mathbb{R}^{n}$ for which there exist $f \in \mathcal{F}$ and $v \in \mathbb{R}^{n}$ such that $f$ is differentiable at $v$ and $\gamma=\nabla f(v)$. Here and throughout, $\nabla f(x)$ denotes the gradient of $f$ at a point $x$ at which $f$ is differentiable.

We now state our main result:

Theorem 2.1. Let $\mathcal{F}$ be a family satisfying the above conditions. The following statements are equivalent:

(a) $\nabla(\mathcal{F})$ is dense in $\mathbb{R}^{n}$.

(b) Any compact convex set, say $K \subset \mathbb{R}^{n}$, is the intersection of all sets of the form $B_{f}+w$, such that $B_{f}+w \supset K, f \in \mathcal{F}$ and $w \in \mathbb{R}^{n}$.

This result may be translated in a slightly different form, more in the geometrical style of Minkowski's theorem language. For, let $B$ be a closed convex set and $v$ a point on the boundary of $B$, at which that boundary is differentiable. Denote by 
$\gamma_{v}$ the only element of $\mathbb{R}^{n}$ satisfying the conditions $\left\|\gamma_{v}\right\|=1$ and $\left\langle\gamma_{v} \mid x-v\right\rangle \leq 0$ for all $x \in B$. So $\gamma_{v}$ is the outward, unit, normal vector to $B$ at $v$. The set of all $\gamma_{v}$, with $v$ running over the set of points of differentiability of the boundary of $B$, will be denoted by $\mathcal{N}_{B}$.

Let $\mathcal{S}$ be a family of closed convex sets, each of which has nonempty interior; assume $\mathcal{S}$ is closed for translations and positive homotheties, i.e.

$$
\alpha B+w \in \mathcal{S} \text { for any } B \in \mathcal{S}, w \in \mathbb{R}^{n} \text { and } \alpha>0 .
$$

Define

$$
\mathcal{N}(\mathcal{S}):=\bigcup_{B \in \mathcal{S}} \mathcal{N}_{B}
$$

Theorem 2.2. For a family $\mathcal{S}$ of sets satisfying the above conditions, the following statements are equivalent:

$(\alpha) \mathcal{N}(\mathcal{S})$ is dense in the unit sphere of $\mathbb{R}^{n}$. $\mathcal{S}$.

( $\beta$ ) Any compact convex subset of $\mathbb{R}^{n}$ is the intersection of a subfamily of family

In the following section we prove Theorem 2.1. Theorem 2.2 is a simple consequence. To see that, let us pick any $B \in \mathcal{S}$ and let $b$ be any point of $B$. As $B-b$ is a closed convex neighborhood of the origin, the Minkowski gauge of $B-b$, defined by

$$
f_{B, b}(x):=\inf \{\lambda>0: x \in \lambda(B-b)\}
$$

for any $x \in \mathbb{R}^{n}$, is a nonnegative, convex, positively homogeneous functional. Clearly, $B$ is the unit ball of $f_{B, b}$. It is easily seen that the family

$$
\mathcal{F}_{\mathcal{S}}:=\left\{f_{B, b}: B \in \mathcal{S} \text { and } b \in \operatorname{int}(B)\right\}
$$

satisfies the preconditions of Theorem 2.1. Now Theorem 2.2 is nothing but a restatement of Theorem 2.1 applied to $\mathcal{F}_{\mathcal{S}}$.

\section{LEMMAS AND PROOFS}

We adopt here the notation of section 2. In the next two lemmas we fix a functional $f \in \mathcal{F}$ and a point $v$ where $f$ is differentiable and satisfies $f(v)=1$. We again denote by $\gamma$ the gradient of $f$ at $v$; moreover, the symbol $\Gamma$ represents the subspace of $\mathbb{R}^{n}$ orthogonal to $\gamma$. Recall (cf. [5, Theorem 25.1]) that $\gamma$ is the only vector satisfying

$$
f(x)-f(v) \geq\langle\gamma \mid x-v\rangle, \quad \text { for all } x \in \mathbb{R}^{n} .
$$

In particular, we have $\langle\gamma \mid v\rangle=f(v)=1$ and, therefore, $\gamma \neq 0$.

For any positive $\delta$ and $\lambda$, define:

$$
\begin{aligned}
C_{\delta}(\lambda) & :=\{x \in \Gamma: f(x+\lambda v) \leq \lambda+\delta\} \\
& =\Gamma \cap\left[(\lambda+\delta) B_{f}-\lambda v\right] \\
& =\left[(\lambda v+\Gamma) \cap(\lambda+\delta) B_{f}\right]-\lambda v .
\end{aligned}
$$

Lemma 3.1. For positive $\lambda, \delta$ and $\mu$, we have:

(a) In the relative topology of $\Gamma, C_{\delta}(\lambda)$ is a closed, convex neighborhood of the origin.

(b) $C_{\delta}(\lambda+\mu) \supset C_{\delta}(\lambda)$.

(c) $\Gamma=\bigcup_{\lambda>0} C_{\delta}(\lambda)$. 
Proof. (a) and (b) are easy to prove.

(c) Let $z \in \Gamma$. Choose $\epsilon>0$, in such a way that $\epsilon\|z\| \leq \delta$. As $\gamma=\nabla f(v)$, there exists $\eta>0$ such that

$$
f(x+v)-f(v)-\langle\gamma \mid x\rangle \leq \epsilon\|x\|,
$$

for all $x$ satisfying $\|x\|<\eta$. Let us choose $\lambda>0$ such that $\|z\| / \lambda<\eta$. As $\langle\gamma \mid z\rangle=0$, (4) yields:

$$
f\left(\lambda^{-1} z+v\right)-f(v) \leq \epsilon\|z\| / \lambda .
$$

Therefore

$$
f(z+\lambda v) \leq \lambda+\epsilon\|z\| \leq \lambda+\delta .
$$

This means that $z \in C_{\delta}(\lambda)$, and so (c) is proved.

Lemma 3.2. Let $L$ be a bounded subset of $\mathbb{R}^{n}$, such that $\langle\gamma \mid y\rangle \leq 0$ for all $y \in L$. Given $\delta>0$, there exists $\lambda_{0}>0$ such that any $\lambda \geq \lambda_{0}$ satisfies

$$
L+\lambda v \subset(\lambda+\delta) B_{f} .
$$

Proof. Any $y \in \mathbb{R}^{n}$ can be uniquely represented as

$$
y=x-\mu v, \quad \text { where } x \in \Gamma \text { and } \mu \in \mathbb{R} .
$$

In case $y \in L$, the corresponding $\mu$ is obviously nonnegative. As $L$ is bounded, there exist $r, s \in \mathbb{R}$ such that, for any $y \in L$, the representation (5) satisfies

$$
\|x\| \leq s \quad \text { and } \quad 0 \leq \mu \leq r .
$$

By Lemma 3.1(b)-(c), there exists $\lambda_{0}>0$ such that, for any $\lambda \geq \lambda_{0}$ :

$$
\begin{aligned}
& \lambda \geq 2 r, \\
& C_{\delta}(\lambda) \supset\{z \in \Gamma:\|z\| \leq 2 s\} .
\end{aligned}
$$

Let $y \in L$. It follows from (5)-(7) that the vector

$$
z:=\frac{\lambda}{\lambda-\mu} x
$$

satisfies

$$
\|z\| \leq \frac{\lambda s}{\lambda-r} \leq 2 s .
$$

By (8), $z \in C_{\delta}(\lambda)$. Therefore $f(z+\lambda v) \leq \lambda+\delta$. Finally, the inequality

$$
f(y+\lambda v)=\frac{\lambda-\mu}{\lambda} f(z+\lambda v) \leq \lambda+\delta
$$

proves $y+\lambda v \in(\lambda+\delta) B_{f}$.

Proof of Theorem 2.1. (a) $\Rightarrow$ (b) Let us fix a point $u \notin K$. There exists a hyperplane which strictly separates $u$ and $K$; this means that there exist $a$ and $\gamma$ in $\mathbb{R}^{n}$, such that

$$
\begin{aligned}
& \langle\gamma \mid u-a\rangle>0, \\
& \langle\gamma \mid x-a\rangle<0, \text { for any } x \in K .
\end{aligned}
$$

The set of all $\gamma$ 's satisfying (9)-(10) (for a fixed $a$ ) is open, because $K$ is compact. Therefore, as we are assuming (a), there exists $\gamma \in \nabla(\mathcal{F})$ satisfying (9)-(10). Accordingly, let $v \in \mathbb{R}^{n}$ and $f \in \mathcal{F}$ be such that $f(v)=1, f$ differentiable at $v$ and $\nabla f(v)=\gamma$. 
Obviously, $f$ is differentiable at $\tau v$, for any positive $\tau$, and $\gamma=\nabla f(\tau v)$. Therefore, for $x \in \mathbb{R}^{n}$ :

$$
f(x) \geq f(\tau v)+\langle\gamma \mid x-\tau v\rangle=\tau+\langle\gamma \mid x-\tau v\rangle .
$$

Choose $\delta>0$ in such a way that

$$
\langle\gamma \mid u-a\rangle>\delta\langle\gamma \mid v\rangle \text {. }
$$

If we let $x:=u-a+\lambda v$ and $\tau:=\lambda+\delta$, where $\lambda$ is an arbitrary positive real number, we obtain from (11):

$$
f(u-a+\lambda v) \geq \lambda+\delta+\langle\gamma \mid u-a-\delta v\rangle>\lambda+\delta .
$$

This means that, for any $\lambda>0$ :

$$
u \notin(\lambda+\delta) B_{f}+a-\lambda v .
$$

By Lemma 3.2 applied to $L:=K-a$, there exists a positive $\lambda_{0}$ such that $K-a+$ $\lambda_{0} v \subset\left(\lambda_{0}+\delta\right) B_{f}$, i.e.

$$
K \subset\left(\lambda_{0}+\delta\right) B_{f}+a-\lambda_{0} v .
$$

The relations (13)-(14) show that $\left(\lambda_{0}+\delta\right) B_{f}+a-\lambda_{0} v$ separates $u$ and $K$, and so (b) is proved.

(b) $\Rightarrow$ (a). We shall apply (b) with $K=B_{e}$, the Euclidean unit ball. Fix $\epsilon>0$ and $u \in \mathbb{R}^{n}$ such that $\|u\|=1$. By (b), there exist $f \in \mathcal{F}$ and $w \in \mathbb{R}^{n}$ such that $B_{f}+w$ separates $(1+\epsilon) u$ from $B_{e}$, i.e. one has

$$
\begin{aligned}
& f(x-w) \leq 1, \text { for } x \in B_{e}, \\
& f((1+\epsilon) u-w)>1 .
\end{aligned}
$$

Define $z:=(1+\epsilon) u-w$. Let $\zeta$ be any element of $\partial f(z)$, the subdifferential of $f$ at $z$ (see $\left[5\right.$, Part V]). For each $x \in \mathbb{R}^{n}$ we have:

$$
f(x)-f(z) \geq\langle\zeta \mid x-z\rangle .
$$

Combining (15)-(17) we get, for all $x \in \mathbb{R}^{n}$ :

$$
f(x-w)-1>\langle\zeta \mid x-(1+\epsilon) u\rangle .
$$

Therefore, $\langle\zeta \mid x-(1+\epsilon) u\rangle<0$, for all $x \in B_{e}$. Applying this to $x:=\|\zeta\|^{-1} \zeta$ we obtain

$$
\left\langle\|\zeta\|^{-1} \zeta \mid u\right\rangle>\frac{1}{1+\epsilon}>1-\epsilon .
$$

Hence, we may draw the following conclusion:

$$
\left\|\frac{\zeta}{\|\zeta\|}-u\right\|<\sqrt{2 \epsilon}, \quad \text { for any } \zeta \in \partial f(z) .
$$

On the other hand (cf. [5, Theorem 25.5]), we know that the set of points where $f$ is differentiable is dense in $\mathbb{R}^{n}$. Moreover (cf. [5, Theorem 25.6]), if $\left(z_{m}\right)$ is a sequence of points where $f$ is differentiable, and $\left(z_{m}\right)$ converges to $z$, then

$$
\lim _{m \rightarrow \infty} \nabla f\left(z_{m}\right) \in \partial f(z) .
$$

Therefore there exists $\zeta_{0} \in \partial f(z)$ and $k \in \mathbb{N}$, such that the vector $c:=\nabla f\left(z_{k}\right)$ is nonzero and

$$
\left\|\frac{\zeta_{0}}{\left\|\zeta_{0}\right\|}-\frac{c}{\|c\|}\right\|<\epsilon
$$


The inequalities (18) and (19) yield

$$
\left\|u-\frac{c}{\|c\|}\right\|<\sqrt{2 \epsilon}+\epsilon .
$$

Our assumption (3) implies $\|c\|^{-1} f \in \mathcal{F}$. It is also clear that

$$
\|c\|^{-1} c=\nabla\left(\|c\|^{-1} f\right)\left(z_{k}\right) .
$$

Therefore $\|c\|^{-1} c \in \nabla(\mathcal{F})$, and so (20) proves item (a) of our theorem.

\section{ACKNOWLEDGEMENT}

We are indebted to the referee for this article's title.

\section{REFERENCES}

1. V.L. Klee, Extremal Structure of Convex Sets II, Math. Z. 69(1958), 90-104 MR 19:1065b

2. S.R. Lay, On Separation by Spherical Surfaces, Amer. Math. Monthly 78(1971), 1112-1113 MR 45:9248

3. S.R. Lay, Separation by Cylindrical Surfaces, Proc. Amer. Math. Soc. 36(1972), 224-228 MR 46:9865

4. S.R. Lay, Separating two Compact Sets by a Parallelotope, Proc. Amer. Math. Soc. 79(1980), 279-284 MR 81d:52005

5. R.T. Rockafellar, Convex Analysis, Princeton Univ. Press, Princeton 1970 MR 43:445

Departamento de Matemática, Universidade de Coimbra, 3000 Coimbra, Portugal

E-mail address: jfqueiro@gemini.ci.uc.pt 